\title{
Role of multi-layer pyramidal truss cores as an inner material of energy absorbing structures
}

\author{
Sangwoo Kim ${ }^{\mathrm{a}}$ \\ Jong-Su Kang ${ }^{b}$ \\ Jae-Yong Lim* \\ a Materials Deformation Department, Korea Institute of Materials Science, Changwon, Gyeongnam, 51508, Republic of Korea. \\ E-mail: kimsw@kims.re.kr \\ ${ }^{b}$ Reliability \& Safety R\&D Division, Extreme Technology R\&D Center, Korea Automotive Technology Institute, Cheonan-si, Chungnam, \\ 31214, Republic of Korea. E-mail: kangjs@katech.re.kr \\ c Department of Safety Engineering, Seoul National University of Science and Technology, Seoul, 01811, Republic of Korea. \\ E-mail: jaeyong.lim@gmail.com \\ * Corresponding author
}

http://dx.doi.org/10.1590/1679-78255611

\begin{abstract}
In this study, finite element analysis was performed to investigate the feasibility of multi-layered pyramidal truss structures as a filler material of energy absorbing tubes. Rectangular tubes with the filler and empty tubes were compressed at a constant velocity of $10 \mathrm{~km} / \mathrm{h}$ and their energy absorbing capabilities were compared to demonstrate the structural benefit of filling materials in the tubes. Additionally, the compressive response of the multi-layered pyramidal truss structures without tube wall constraint were observed. The investigations included three tube wall thicknesses and three pyramidal truss structures with three relative densities by varying the inclination angle of the pyramidal truss strut. Those were made of Al6063T5 and 304 stainless steel (SS304) for tube wall and pyramidal truss structure, respectively. The results indicate that the energy absorption capability of the tube with the filling exceeds the simple sum of that of tube and that of the filler, and this is due to the interaction effect between outer tubes and pyramidal truss struts near the tube wall. Furthermore, the inclination angle of pyramidal truss struts influences energy absorption. Thus, the pyramidal truss structures can be potentially applied as a filler material for energy absorbing structures.
\end{abstract}

\section{Keywords}

multi-layered truss structures, crash box, filler material, Finite Element Analysis, energy absorption.

\section{INTRODUCTION}

Passive energy dissipation devices absorb a portion of energy transmitted to passengers from vehicle collisions. For example, crash boxes, installed behind bumpers, fold progressively when exposed to unexpected impact loads. Dynamic progressive buckling is the primary deformation mechanism of the energy absorbers and leads to oscillations about a reasonably constant operating force over a relatively long distance.

Generally, they are in the shape of rectangular or circular tubes in automobile applications. However, energies are absorbed more effectively with less materials if a supplemental material, such as metal foams, is filled in the empty region of tubes. Thus, the mean crushing strength of the tube filled with metal foam significantly exceeds the simple sum of the energy absorptions of individual elements: the outer tubes and the filler material. Extant studies indicate that interactions between the outer tubes and inner material lead to the additional enhancements (Santosa and Wierzbicki, 1998; Seitzberger et al., 1997; Ashby et al., 2000). 
To date, a significant amount of studies focused on discovering the energy absorption characteristics of empty tubes. The collapse modes of axially compressed tubes are significantly influenced by their cross sections. Abramowicz and Jones (1986) conducted an experimental study on circular and square steel tubes and observed their compressive response. In order to predict the energy absorbing capabilities and buckling wavelengths, Wierzbicki and Abramowicz (1983) performed an analytical study on the progressive buckling of circular tubes. The development was based on the assumptions wherein a rigid-plastic material allowing limited extensional modes. Recently, Velmururugan and Muralikannan (2009) experimentally explored empty tubes of various cross sections and compared their results with simple analytical predictions. In addition to basic geometries including rectangular, square and circular cross sections, double-walled and multi-cell tubes were also tested for energy absorbing performance (Abramowicz and Wierzbicki, 1988; Goel, 2015; Karagiozova, 2004; Kim, 2002; Santosa and Wierzbicki, 1999; Yin et al., 2014). The parent materials comprising of the tubes as well as cross sectional shapes are of interest. Fundamental material response, such as strain hardening, strain rate sensitivity, and fracture mode, evidently influences the collapse modes and energy absorbing mechanisms of the structure (Ronchietto et al., 2009), and thus the tubes made of annealed steel (Velmururugan and Muralikannan, 2009), extruded aluminum alloy (Goel, 2015), composite (Harte et al.. 2000; Yan et al., 2014; Supian et al., 2018) and metal-FRP hybrid (Kalhor and Case, 2015) were explored.

In order to improve the energy absorbing performance, the structural benefit and weight saving were investigated by inserting a lightweight material inside empty tubes (Abramowicz and Wierzbicki, 1988). For example, earlier studies (Hanssen et al., 2000; Seitzberger et al., 2000; Santosa et al., 2000; Shahbeyk et al., 2005) considered aluminum foams. When the foam-filled structures were crushed, it revealed that the mean crushing load significantly increased due to the interaction between the tube wall and filler material. Therefore, the mean crushing strength of the tube filled with metal foam significantly exceeds the simple sum of the energy absorptions of individual elements, namely the outer tube and filler material. In addition to the Al foams, diverse lightweight materials such as polyurethane foam (Yan et al., 2014; Zhang et al., 2014), honeycomb (D'Mello and Wass, 2012; Zaeri and Kroger, 2008), and functionally graded materials (FGM) (Sun et al.,2010) are inserted to investigate their structural performances. Among the lightweight materials, recent studies focused on periodic cellular metals (PCMs). A significant amount of previous studies underlined their structural advantages as well as multifunctional potentials. Furthermore, it is easy to manipulate their mechanical properties simply by altering geometric dimensions. Given their advantages over metal foams (such as structural performance and multifunctional capabilities), the structured materials focused on a potential replacement for metal foam (Wadley, 2006).

In the present study, a multi-layered pyramidal truss structure that corresponds to periodic cellular metal (PCM), is introduced for a filler material of crash boxes. A certain amount of crushing force enhancement is expected when a multilayered truss structure is inserted into an empty tube and subsequently compressed. Thus, the present study performed numerical simulations in order to understand the deformation mechanism and quantify the amount of interactions between the outer tube and multi-layered pyramidal truss structures (PCMs). Additionally, the compressive responses of individual elements which correspond to empty tubes and multi-layered pyramidal truss structures are also investigated to analyze energy absorbing capacity.

The organization of this study is as follows. In section 2, three types of compression models are constructed as follows: (i) empty Al6063-T5 rectangular tubes, (ii) multi-layered pyramidal truss structures (PCM), and (iii) PCM filled Al6063-T5 tubes. In Section 3, three types of numerical simulation results are presented and discussed.

\section{NUMERICAL ANALYSIS}

\subsection{Energy absorbing tubular structure}

The present study investigates a PCM-filled tubular structure consisting of an outer tube and an inner material as schematically described in Figure 1(a). A tube with a height of $160 \mathrm{~mm}$ exhibits a rectangular cross section with a width (W) corresponding to $100 \mathrm{~mm}$ and a length (L) corresponding to $60 \mathrm{~mm}$.

The base material for the outer tube is Al6063-T5, and this is currently employed in automotive crash boxes. Three wall thicknesses of $h=1.0,1.2,1.5 \mathrm{~mm}$ are considered. Given a constant cross section along the height direction, extrusion is one of the easiest manufacturing methods for the outer tubes, and thus the base material is determined as mentioned above.

A multi-layered pyramidal truss structure made of 304 stainless steel (SS304) is considered as a filler material. Previous studies report that the SS304 pyramidal truss structures are mainly manufactured by perforating/bending and then joined with face sheets by brazing for sandwich construction. Given the periodic nature of the multi-layered pyramidal truss structure, a representative unit cell is introduced for characterization as shown in Figure 1(b). The width of an unit cell $\left(W_{c}\right)$ the angle of strut to the base plane $(\alpha)$, and strut thickness $\left(t_{c}\right)$ are used, and the strut is assumed to 
have a square cross-section. And, the nodal region of the pyramidal truss structure, is assumed to have a flat area $\left(b_{c}{ }^{\prime}\right.$ $\left.b_{c}\right)$ for brazing neighboring upper and lower cells. Those dimensions are associated with the relative density $\left(\bar{\rho}_{c}\right)$, which is defined as the ratio of the density of pyramidal truss structures to that of base material. The investigations were performed on several pyramidal truss structures with three relative densities by varying angle $\alpha$ from $30^{\circ}$ to $40^{\circ}$ while fixing $t_{c}=1.0 \mathrm{~mm}, b_{c}=1.414 \mathrm{~mm}$ and $W_{c}=12 \mathrm{~mm}$. In this case, the relative density is obtained by using Eqs. (1) and (2) as follows:

$$
\bar{\rho}_{c} \approx \frac{\left(2 b_{c}^{2} t_{c}+4 l_{c} t_{c}\right)}{\left(\sqrt{2} l_{c} \cos \alpha+2 b_{c}\right)\left(\sqrt{2} l_{c} \cos \alpha+2 b_{c}\right)\left(l_{c} \sin \alpha+t_{c}\right)}
$$

$2 \sqrt{2} b_{c}+2 l_{c} \cos \alpha=\sqrt{2} W_{c}$

This is shown in Figure 1. In addition to the above-mentioned integrated structure, empty aluminum tubes without inner materials are compressed and compared with the compressive response of PCM-filled tubes. Furthermore, the compression response of multi-layered pyramidal truss structures is also characterized. Table 1 lists all the numerical models used in the study.

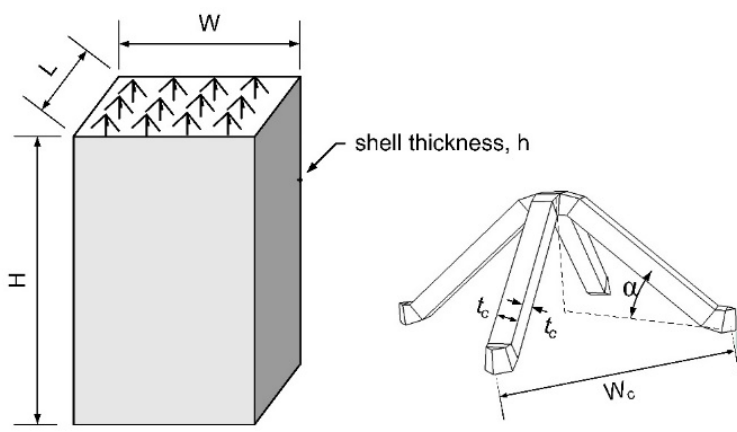

(A)

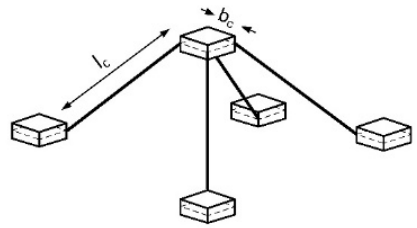

(B)

Figure 1. (a) schematic diagram of the outer tube (Al6063-T5) and multi-layered pyramidal truss structure inside; (b) a representative unit cell of multi-layered PCM structure and its simplification.

Table 1. List of FE models

\begin{tabular}{|c|c|c|c|c|c|c|c|c|c|c|}
\hline \multirow{2}{*}{$\begin{array}{l}\text { Case } \\
\text { No. }\end{array}$} & \multicolumn{4}{|c|}{ (Outer Shell, Al6063T5) } & \multicolumn{5}{|c|}{ (Inner Core) } & \multirow[b]{2}{*}{ comment } \\
\hline & $\mathbf{w}$ & $\mathbf{L}$ & $\mathbf{H}$ & $\mathbf{h}$ & $\begin{array}{l}\text { Wc' } \\
\text { Wc }\end{array}$ & $\begin{array}{l}\mathrm{tc}^{\prime} \\
\mathrm{tc}\end{array}$ & $\alpha c$ (deg) & $\begin{array}{l}\text { bc'bc } \\
\text { (node) }\end{array}$ & $\bar{\rho}$ & \\
\hline$\# 1$ & 100 & 60 & 160 & 1.0 & $12^{\prime} 12$ & $1^{\prime} 1$ & 30 & $1.414^{\prime} 1.414$ & 0.0497 & PCM-filled crashbox (Model type III) \\
\hline$\# 2$ & 100 & 60 & 160 & 1.0 & $12^{\prime} 12$ & $1^{\prime} 1$ & 35 & $1.414^{\prime} 1.414$ & 0.0447 & PCM-filled crashbox (Model type III) \\
\hline$\# 3$ & 100 & 60 & 160 & 1.0 & $12^{\prime} 12$ & $1^{\prime} 1$ & 40 & $1.414^{\prime} 1.414$ & 0.0408 & PCM-filled crashbox (Model type III) \\
\hline$\# 4$ & 100 & 60 & 160 & 1.2 & $12^{\prime} 12$ & $1^{\prime} 1$ & 30 & $1.414^{\prime} 1.414$ & 0.0497 & PCM-filled crashbox (Model type III) \\
\hline$\# 5$ & 100 & 60 & 160 & 1.2 & $12^{\prime} 12$ & $1^{\prime} 1$ & 35 & $1.414^{\prime} 1.414$ & 0.0447 & PCM-filled crashbox (Model type III) \\
\hline \#6 & 100 & 60 & 160 & 1.2 & $12^{\prime} 12$ & $1^{\prime} 1$ & 40 & $1.414^{\prime} 1.414$ & 0.0408 & PCM-filled crashbox (Model type III) \\
\hline$\# 7$ & 100 & 60 & 160 & 1.5 & $12^{\prime} 12$ & $1^{\prime} 1$ & 30 & $1.414^{\prime} 1.414$ & 0.0497 & PCM-filled crashbox (Model type III) \\
\hline$\# 8$ & 100 & 60 & 160 & 1.5 & $12^{\prime} 12$ & $1^{\prime} 1$ & 35 & $1.414^{\prime} 1.414$ & 0.0447 & PCM-filled crashbox (Model type III) \\
\hline \#9 & 100 & 60 & 160 & 1.5 & $12^{\prime} 12$ & $1^{\prime} 1$ & 40 & $1.414^{\prime} 1.414$ & 0.0408 & PCM-filled crashbox (Model type III) \\
\hline$\# 10$ & 100 & 60 & 160 & 1.0 & - & - & - & - & - & Empty tube (Model type I) \\
\hline$\# 11$ & 100 & 60 & 160 & 1.2 & - & - & - & - & - & Empty tube (Model type I) \\
\hline$\# 12$ & 100 & 60 & 160 & 1.5 & - & - & - & - & - & Empty tube (Model type I) \\
\hline$\# 13$ & - & - & - & - & $12^{\prime} 12$ & $1^{\prime} 1$ & 30 & $1.414^{\prime} 1.414$ & 0.0497 & $\begin{array}{l}\text { Multi-layered pyramidal truss } \\
\text { (Model type II) }\end{array}$ \\
\hline$\# 14$ & - & - & - & - & $12^{\prime} 12$ & $1^{\prime} 1$ & 35 & $1.414^{\prime} 1.414$ & 0.0447 & $\begin{array}{l}\text { Multi-layered pyramidal truss } \\
\text { (Model type II) }\end{array}$ \\
\hline$\# 15$ & - & - & - & - & $12^{\prime} 12$ & $1^{\prime} 1$ & 40 & $1.414^{\prime} 1.414$ & 0.0408 & $\begin{array}{l}\text { Multi-layered pyramidal truss } \\
\text { (Model type II) }\end{array}$ \\
\hline
\end{tabular}




\subsection{FE model constructions}

As mentioned previously, the following three types of numerical models are constructed: (i) Type I: empty rectangular tubes, (ii) Type II: multi-layered pyramidal truss structures, and (iii) Type III: PCM-filled tubes. Model types I and II correspond to the individual elements of PCM-filled tubes (model type III), outer tube, and filler material (see Table 1). The objective of type I and type II involves analyzing the contribution of energy absorptions and measuring the load enhancement from interactions between the outer tube and the filler material. A commercial FE package, ABAQUS/Explicit, is utilized to simulate the constructed models.

The base materials for outer tubes and multi-layered pyramidal truss structures correspond to AI6063T5 and 304 stainless steel (SS304), respectively. In order to incoporate their material response into FE models, tensile test specimens are extracted from an Al6063T5 extruded tube and a tensile test is performed (KS B 0802, 2013). On the other hand, the material properties of 304 stainless steel (SS304) are based on previous studies (Lim and Bart-Smith, 2014). Specifically, Al6063-T5 is considered as an elastic-plastic solid with an elastic modulus $\mathrm{E}=70513.8 \mathrm{MPa}$, Poisson's ratio v=0.33, density, $\rho=2700 \mathrm{~kg} / \mathrm{m}^{3}$, and yield stress $\sigma_{\gamma}=220.0 \mathrm{MPa}$. The plastic deformation of the aluminum alloy is described by an isotropic hardening model utilizing multiple test data points. On the other hand, 304 stainless steel (SS304) is represented by Young's modulus, yield stress, and the two hardening moduli for the two plastic regions corresponding to: $E=230769.2$ $\mathrm{MPa}, \sigma_{Y}^{(1)}=271.8 \mathrm{MPa}, \sigma_{Y}^{(2)}=450.0 \mathrm{MPa}, E_{T}^{(1)}=7842.0 \mathrm{MPa}, E_{T}^{(2)}=2434.7 \mathrm{MPa}$, respectively. Figure 2 shows the stress-strain curves for both metals. Most of aluminum alloys do not show appreciable strain rate sensitivity, and thus the rate sensitivity of Al6063-T5 is ignored. In contrast, the rate dependency of SS304, (i.e., the base material of multi-layered truss structures) is considered by using the Cowper-Symonds model. The dynamic yield strength enhancement ratio, $R$, is given by

$R=\frac{\sigma_{y}(\dot{p})}{\sigma_{y}^{0}}=1+\left(\frac{\dot{p}}{D}\right)^{m}$

The material parameters of 304 stainless steel (SS304) in Eqn. (3), namely D and $m$, are used as follows: $D=4920$, m=0.154 (Ferri et al., 2006; Lim and Bart-Smith, 2014; Stout and Follansbee, 1986; Zok et al., 2005).

In all the FE models, shell elements and beam elements are used to model the outer tubes and multi-layered pyramidal truss structures, respectively. To guarantee the FE models free from discretization, appropriate mesh density was determined. Several models having several mesh densities were created by doubling the number of elements, and the internal energy results were compared. A mesh density resulting in less than $1 \%$ difference between successive models was chosen to be the converged one. For the outer tubes, the number of shell elements (S4R in ABAQUS) correspond to fifty in the width, thirty in the length and eighty in the height directions, respectively. Furthermore, twenty beam elements are used to model a strut of a pyramidal truss. Thus, approximately 110,000 beam elements are meshed for the multi-layered pyramidal truss structures with $\alpha=35^{\circ}$.

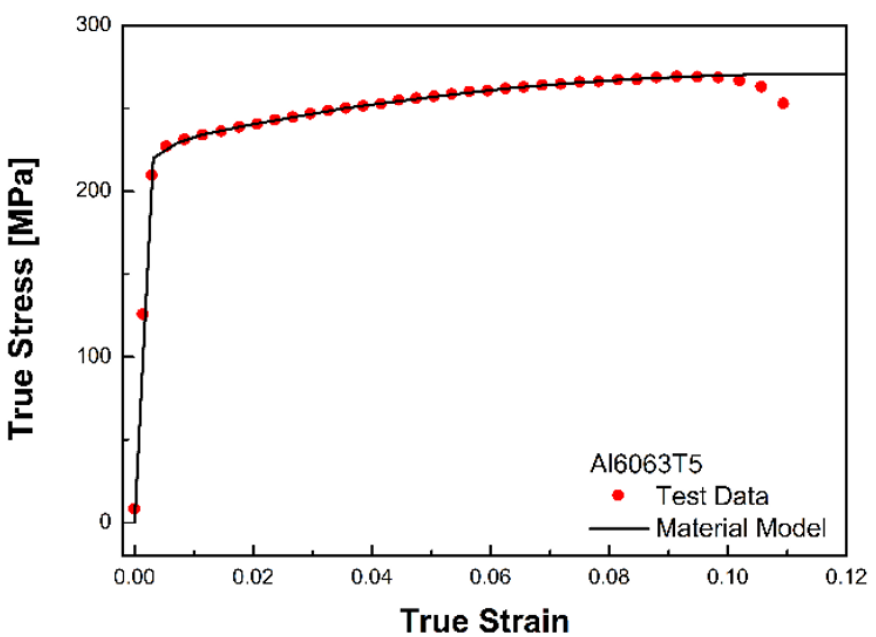

(a)

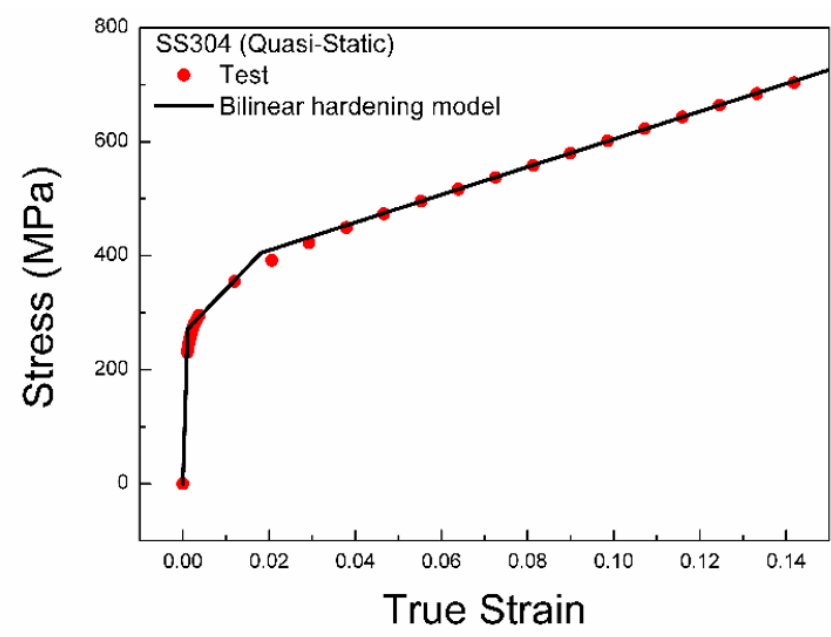

(b)

Figure 2. Stress-strain curves of (a) Al6063-T5; (b) 304 stainless steel (SS304).

Prior to a dynamic compressive analysis, additional eigen-analysis on the outer tube is performed to obtain an imperfect geometry by superposing eigenmodes to a perfectly modelled geometry. The appropriate curvature shape and its magnitude are determined from an additional compression test of an Al6063-T5 empty tube (The details about the 
test are skipped here). The test result is compared, and imperfect geometry from the second eigenmode and its magnitude of $\xi_{2}=0.1$ is selected such that it exhibits a deformation shape and an initial peak in the reaction force curve close to the test results. The determined curvature is applied to all the empty tube models and PCM-filled tube models. This can trigger the formation of buckling and exhibit a more realistic initial peak in reaction-force curves.

Two rigid plates are tied to both the ends of the structure, and dynamic compression is applied to a rigid plate. The plate moves at a constant velocity of $10 \mathrm{kph}(2778 \mathrm{~mm} / \mathrm{s})$ while the other end is completely fixed. With respect to a number of contact interactions, a general contact algorithm without friction is defined between the pyramidal truss struts and a tube, and also from the self-contact between the tube and pyramidal truss struts. Calculations are completed when the amount of compression reaches $50 \%$ of the height of tube (i.e., $80 \mathrm{~mm}$ ).

Additionally, FE models for individual elements comprising of the PCM-filled structures are constructed and simulated in the same manner as mentioned above (i.e., type I and II models). Three empty tubes (type I) with three shell thicknesses $(\mathrm{h})$ are modelled and compressed with the same boundary and loading conditions. Furthermore, with respect to the multi-layered pyramidal truss structure models (type II), three models are constructed by controlling angle, $\alpha$, which varies the relative density. The nodes at the bottom layer and those at the top layer are fixed to two rigid plates wherein one corresponds to fixed and the other corresponds to dynamic compression. All the simulation models, empty tubes (\#10-\#12), multi-layered pyramidal truss structures (\#13-15), and PCM-filled tubes (\#1-\#9) are summarized in Table 1.

\section{RESULTS AND DISCUSSION}

\subsection{Al6063-T5 empty tubes (Type I)}

Three tubes of $\mathrm{h}=1.0,1.2,1.5 \mathrm{~mm}$ are compressed at a constant velocity of $\mathrm{V}=10 \mathrm{~km} / \mathrm{h}(2778 \mathrm{~mm} / \mathrm{s})$. Figure 3 shows the deformation patterns of empty tubes of $h=1.5 \mathrm{~mm}$. Figure 4 shows a reaction force-time curve of empty tubes of $\mathrm{h}=1.0,1.2,1.5 \mathrm{~mm}$. At the very initial stage, the reaction force rises instantaneously. After an initial peak, the reaction force decays and increases again at approximately $d=40 \mathrm{~mm}$. The load fluctuation is clearly associated with the formation of consecutive folds. Similarly, progressive buckling response is also observed in the empty tube of $\mathrm{h}=1.0 \mathrm{~mm}$ and $1.2 \mathrm{~mm}$.

The mean crushing force is calculated by averaging the sum of elastic energy and plastic dissipation over compressive displacement $(\Delta=80 \mathrm{~mm})$.

$P_{m}=\frac{\int_{0}^{\Delta} P \cdot d x}{\Delta}$

In the present study, the FE calculations are compared with the theoretical predictions (Velmururugan and Muralikannan, 2009) as shown in Table 2. The expression is as follows:

$$
P_{m}=38.27 M_{0}\left(\frac{C}{h}\right)^{1 / 3} \text { with } C=\left(\frac{c+d}{2}\right)
$$

The discrepancies between the two are about $10 \%$ are due to the exclusion of the strain hardening response in the FEA.

Table 2 Comparison of mean crushing load, Pm.

\begin{tabular}{cccc}
\hline $\begin{array}{c}\text { Shell thickness, } h \\
(\mathbf{m m})\end{array}$ & $\begin{array}{c}\text { FEA }\left(\mathbf{P}_{\mathbf{m}}\right) \\
(\mathbf{N})\end{array}$ & $\begin{array}{c}\text { Theoretical }\left(\mathbf{P}_{\mathbf{m}}\right) \\
(\mathbf{N})\end{array}$ & $\begin{array}{c}\text { Discrepancy } \\
(\%)\end{array}$ \\
\hline 1.0 & 11428 & 10100 & 13.2 \\
1.2 & 13896 & 13686 & 1.54 \\
1.5 & 22018 & 19852 & 10.9 \\
\hline
\end{tabular}




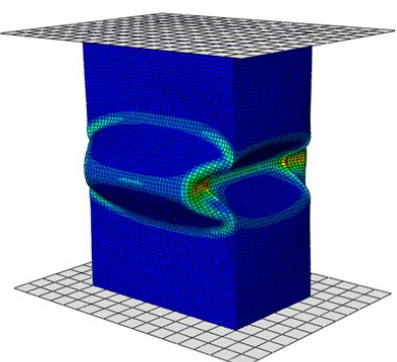

(a)

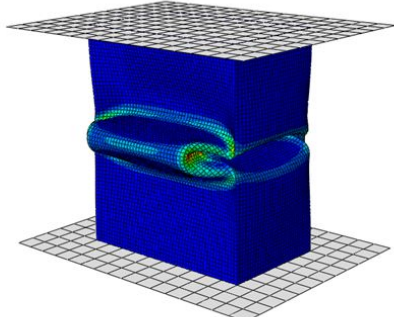

(b)

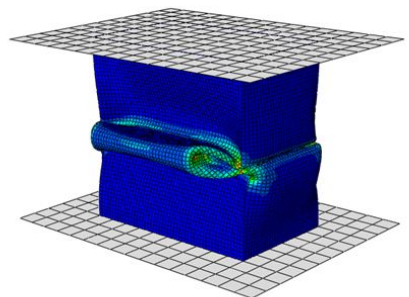

(c)

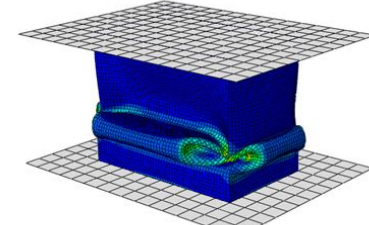

(d)

Figure 3. Deformation pattern of empty tube compression of $h=1.5 \mathrm{~mm}$ under $V=10 \mathrm{~km} / \mathrm{h}$ (a) at $20 \mathrm{~mm}$ compression, (b) at $40 \mathrm{~mm}$ compression, (c) at $60 \mathrm{~mm}$ compression, (d) at $80 \mathrm{~mm}$ compression. The contour represents the equivalent plastic strain.

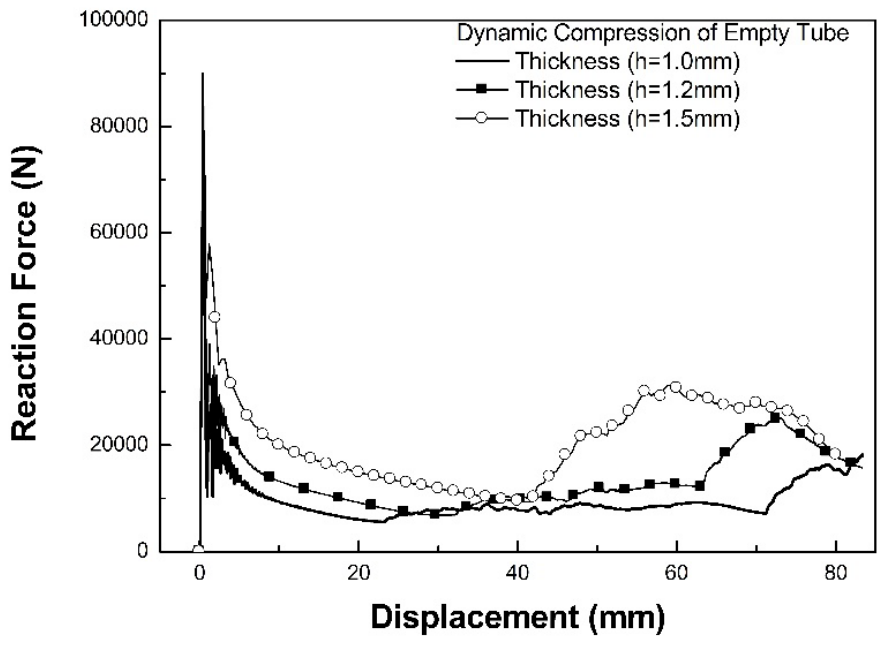

Figure 4. Reaction force time history curve of empty tubes of $h=1.0$ (\#10), 1.2 (\#11), 1.5mm (\#12).

\subsection{SS304 multi-layered pyramidal truss structures (Type II)}

Figure 5 shows a front view of SS304 multi-layered pyramidal truss structure when it is compressed by $40 \mathrm{~mm}$ and $80 \mathrm{~mm}$, respectively. The contour in the figure represents the equivalent plastic strain. Figure 6 shows the reaction force curves with respect to the inclination angle, $\alpha$. At the earliest stage of compression (less than $1 \mathrm{~mm}$ compression), a steep increase in reaction force is observed, and thus is associated with the elastic deformations of individual struts along their own axes. When the curve reaches a point of approximately $4000 \sim 5000 \mathrm{~N}$ (corresponding to a few of mm compression), the rate of the reaction force change begins to decrease. And then, the curve rises at a constant rate as if metallic solids harden beyond the yield strength. In the consdered range of the inclination angle, $\alpha$, differences in the reaction force curves were not appreciable.

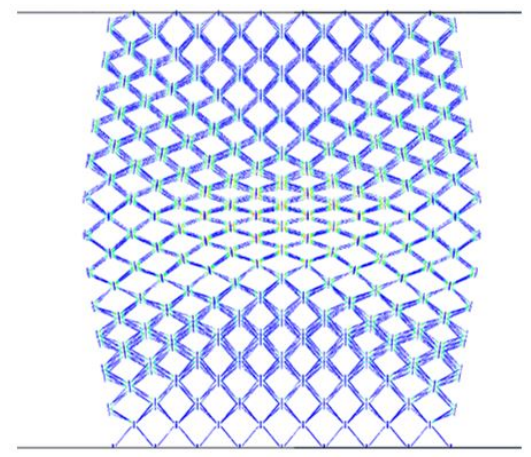

(a)

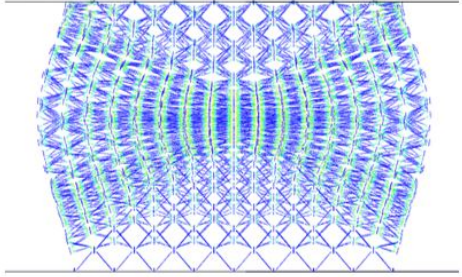

(b)

Figure 5. Compression of the pyramidal truss core at (a) $40 \mathrm{~mm}$ compression ( $25 \%$ its height) and (b) $80 \mathrm{~mm}$ ( $50 \%$ of its height). The contour represents the equivalent plastic strain. 
Two points are noted based on the observations.

The first point is deformation mechanism. In previous studies that investigated the structural response of PCM structures (Cote et al., 2007; Wicks and Hutchinson, 2004; Deshpande et al., 2001), the deformation mechanism is mainly due to axial-stretch of PCM struts as shown in Figure 7. Therefore, when struts are loaded, they are compressed or stretched in their axial direction. Thus, it is well-known that the structural superiority to metal foam stems from the deformation mechanism. However, in the multi-layered pyramidal truss structures, plastic deformations are shown as localized at the nodes where each four struts meet, and this is considered as a 'hinge' deformation mechanism. This is because the multi-layered pyramidal truss structure is manufactured such that a single pyramidal truss layer is repeatedly layered up to a considerable height so that it can be considered as open cell foam with a significantly large pore size.

The second point is that the multi-layered pyramidal truss structures bulge in the middle of their height. In the case of sandwich structures with a single layer of pyramidal truss core, the displacement of nodes is constrained because all the nodes of the truss structure are attached to a face sheet. However, if the truss structures are constructed as multilayered, the nodes at the middle layers can move freely such that the bulging is observed.

Thus, the deformation mechanism makes the load curves independent of a geometric parameter, $\alpha$. The mean crushing load is approximately in the range of $5000-8000 \mathrm{~N}$ during compression up to fifty percent of their heights.

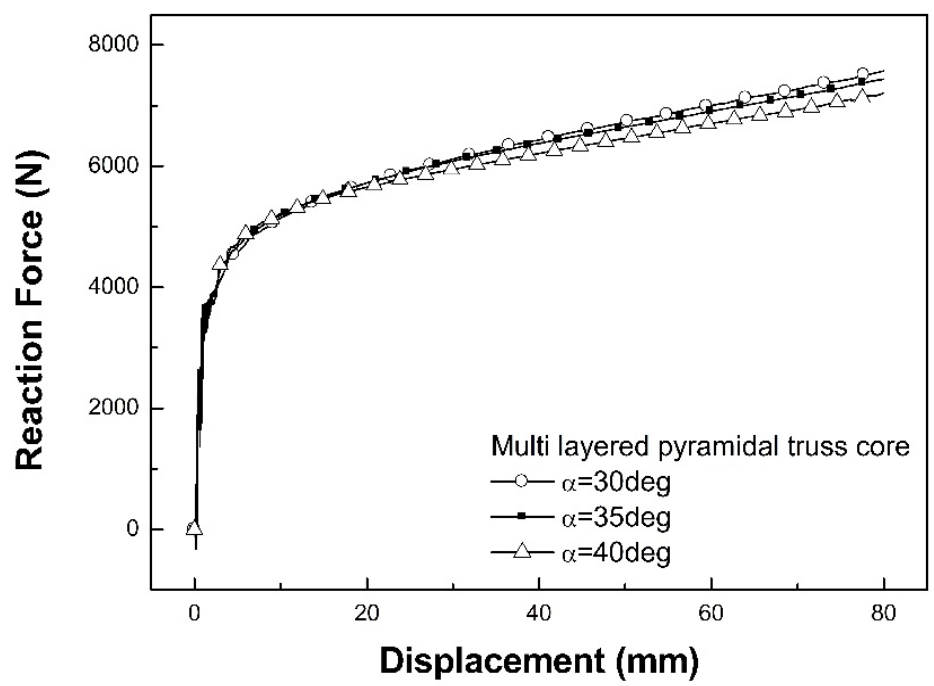

Figure 6. Reaction force-displacement curve of multi-layered pyramidal truss structures (PCM) with respect to $\alpha$.
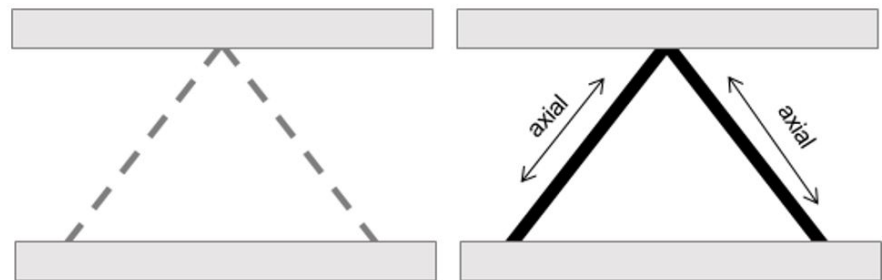

(a) Single layer pyramidal truss core when applied to a sandwich core
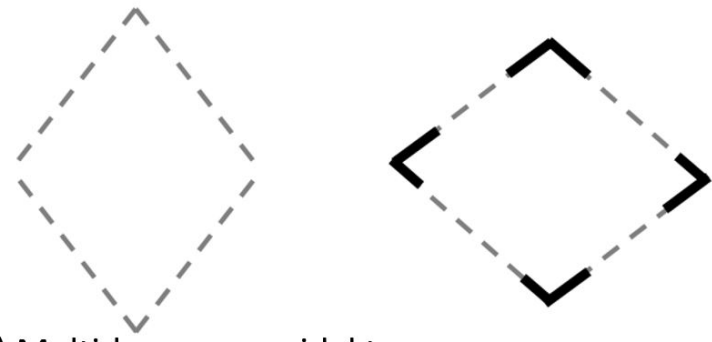

(b) Multi-layer pyramidal truss core

Figure 7. Deformation mechanism of pyramidal truss (dotted: undeformed, solid: deformed) 


\subsection{PCM-filled structures (Type III)}

Figure 8 compares crushing patterns between an empty tube and PCM filled one. When compressed, the outer tubes collapse progressively. However, the bulging is not appreciably observed as seen in the type II analysis because the outer tube constrains the expansion of the inner material. Hence, the struts near an outer tube are observed to severely deform as shown in Figure 8. Figure 9(a) compares the reaction force-displacement curve of PCM-filled tubes with $\mathrm{h}=1.5 \mathrm{~mm}$ and $\alpha=40^{\circ}$, that of an empty tube with $\mathrm{h}=1.5 \mathrm{~mm}$, and that of a multi-layered pyramidal truss with $\alpha=40^{\circ}$. Furthermore, the contribution of plastic dissipation by individual elements is also shown in Figure $9(\mathrm{~b})$. When compared with the mean crushing force of empty tube with $h=1.5 \mathrm{~mm}(22000 \mathrm{~N})$, the load enhancement due to the PCM filler is evident.

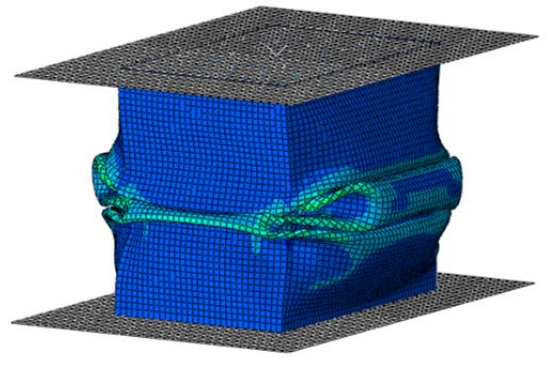

(a)

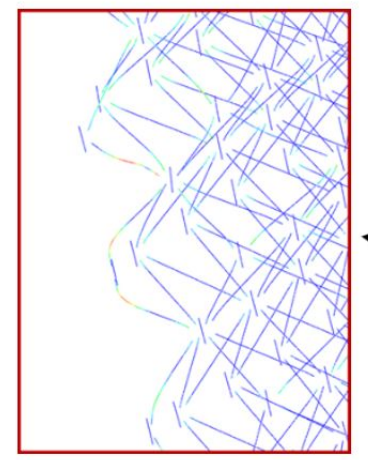

(b)

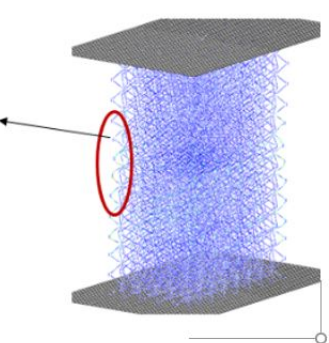

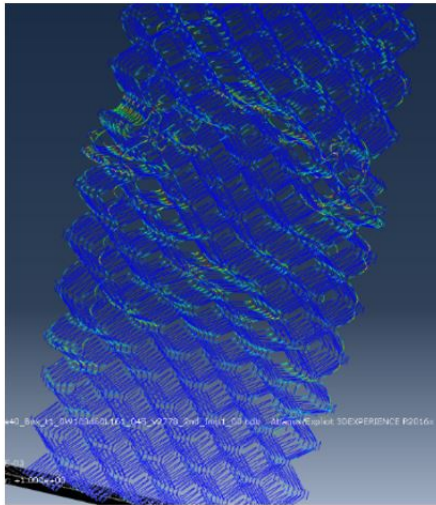

(c)

Figure 8. Deformation patterns of PCM-filled tubes.

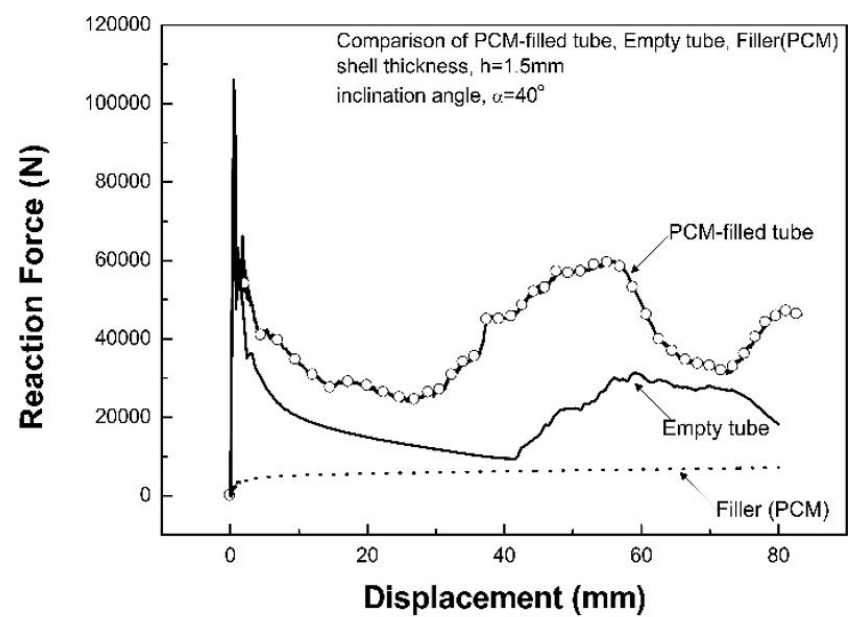

(a)

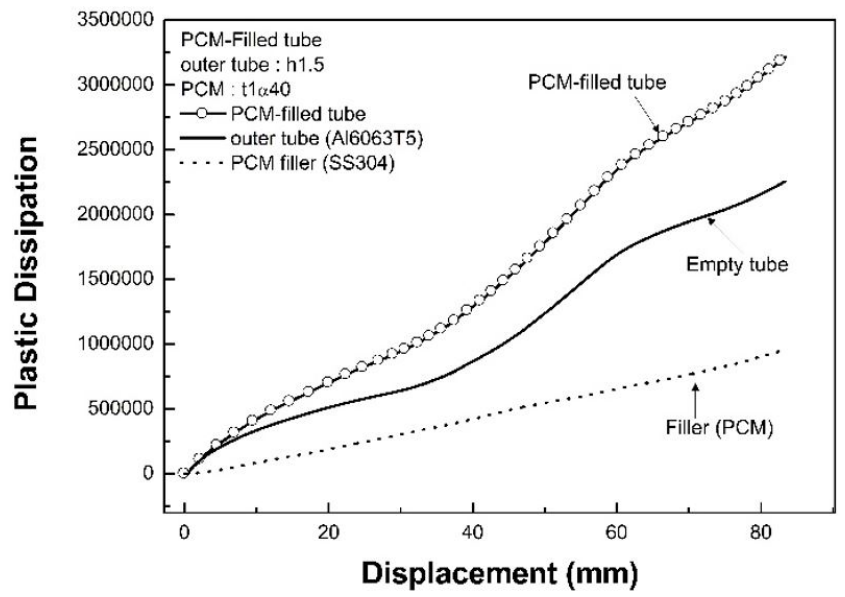

(b)

Figure 9. (a) Comparison of load-displacement curves of an empty tube of $\mathrm{h}=1.5 \mathrm{~mm}$, a multi-layered pyramidal truss structure, and PCM-filled structures; (b) analysis of energy absorptions of a PCM-filled structure $\left(h=1.5, \alpha=40^{\circ}\right)$

Several studies (Seitzberger et al., 1997;Seitzberger et al., 2000; Santosa et al., 2000) observed that foam-filled tubes exhibit buckling wavelengths that are shorter than those of empty tubes with same dimensions, and this result in the enhancement of energy absorption capacity. Conversely, in the present study, the wave lengths in the integrated PCMfilled structures (type III) are observed to slightly exceed those of empty tube simulations (type I). Nevertheless, the energy absorption capacity of integrated structure is greater than the sum of type I and type II models by $20 \sim 50 \%$. The aforementioned structural benefit is strongly associated with the severe deformations of neighboring struts near the tube material, and this corresponds to the interaction between the outer tube material and PCM structures.

Figure 10 quantitatively summarizes the amount of interaction between tube and core based on the angle $\alpha$. As shown in the diagram, the energy absorptions of PCM-filled structures approximately exceeds the simple sum of the calculations from type I and type II analyses by $20 \sim 50 \%$. The $20 \sim 50 \%$ differences are denoted by black arrows as shown in Figure 10. 


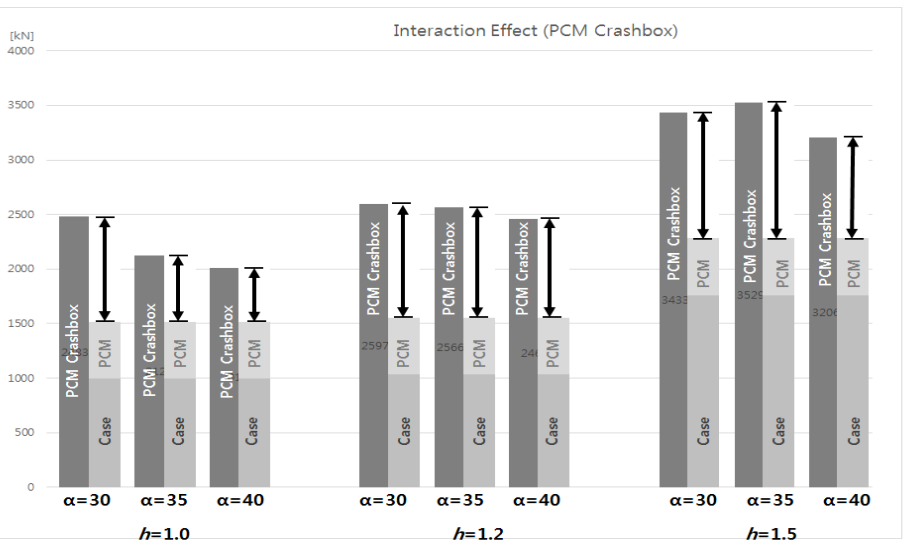

Figure 10. Summary of the structural benefit obtained by inserting a multilayered pyramidal truss structure as an inner material.

Although the relative density does not vary significantly in the range of $30^{\circ}<\alpha<40^{\circ}$ (which corresponds to $\left.\bar{\rho}_{c}=4.08^{\sim} 4.97 \%\right)$, the amount of mean crushing load $\Delta \mathrm{Pm}$, varies substantially depending on the angle of $\alpha$. It is assumed that the main deformation mechanism of pyramidal truss structures switch from bending to axial-stretch since the outer tube constrains the deformation of multi-layered pyramidal truss struts.

Therefore, multi-layered pyramidal truss structures can be beneficially applied to an inner material at the point of lightweight design of energy absorbers. If the design variables are optimized, increased weight saving is expected with reduced materials. Thus, it is expected that energy absorbing performance can be enhanced by inserting a PCM inner material as performed in metal foam-filled structures.

\section{CONCLUSIONS}

In the study, we examined the feasibility of multi-layered pyramidal truss structures as an inner material of filled crashbox without considering the optimization of material distribution. From the numerical simulations of empty tubes, multi-layered truss cores, and PCM-filled tubes, following conclusions were obtained:

1. Given the interaction effect between outer tubes and PCM struts near the tube, the energy absorption capability of the integrated structure exceeds the sum of capabilities of individual components. By inserting PCMs inside the empty tubes, the energy absorption enhancements amount to $20 \sim 50 \%$ of the sum of capacities of individual elements.

2. The energy absorption capability is associated with the angle $\alpha$. Although the considered range of $\alpha$ did not change the relative density appreciably, it considerably influenced the energy absorption characteristics. For example, with the change in truss angle, $\alpha$, from $30^{\circ}$ to $40^{\circ}$ (corresponding relative density difference of $0.89 \%$ ), the mean crush load, $\mathrm{Pm}$, showed the $16 \%$ difference in the tubes of outer shell thickness, $\mathrm{h}=1 \mathrm{~mm}$.

3. The material response of multilayered pyramidal truss structures, when they are not inserted into tubes, appeared as an elastic-plastic hardening metal. Macroscopically, bulging was observed as compressed. And, deformation was concentrated at the nodes from the microscopic viewpoint. It is noteworthy that the observations are not seen when a single layer of pyramidal truss core is employed in sandwich structures.

\section{Acknowldegement}

This work was partly supported by the Materials and Components Technology Development Program program of MOTIE/KEIT [10076429, Design and fabrication of lightweight \& multi-functional PCM(Periodic Cellular Material) having high-strength and heat-dissipation capability]. And, this work was partially supported by the National Research Foundation of Korea (NRF) grant funded by the Korea government (MSIP; Ministry of Science, ICT \& Future Planning, Grant No.: 2017R1C1B5017363). The authors would like to thank the financial support.

Author's Contribuitions: Conceptualization, S W Kim and J Y Lim; Methodology, J S Kang and J Y Lim; Investigation, S W Kim, J S Kang and J Y Lim; Writing- Original Draft; S W Kim; Writing - Review \& Editing, J Y Lim; Funding Acquisition, S W Kim, J S Kang and J Y Lim; Resources, J S Kang and J Y Lim; Supervision, J Y Lim. 


\section{Editor: Marcílio Alves}

\section{References}

Abramowicz, W., Jones, N. (1986). Dynamic progressive buckling of circular and square tubes. International Journal of Impact Engineering 4(4): 243-270.

Abramowicz, W., Wierzbicki, T. (1988). Axial crushing of foam-filled columns. International Journal of Mechanical Sciences 30 , 3-4, 263-271.

Ashby, M.F., Evans, A., Fleck, N.A., Hutchinson, J.W., Wadley, H.N.G., Gibson, L.J. (2000). Metal Foams: A Design Guide, Butterworth-Heinemann (Oxford).

Cote, F., Biagi, R., Bart-Smith, H., Deshpande, V.S. (2007). Structural response of pyramidal core sandwich columns. International Journal of Solids and Structures 44: 3533-3556.

Deshpande, V.S., Ashby, M.F., Fleck, N.A. (2001). Foam topology: bending versus stretching dominated architectures. Acta Materialia 49: 1035-1040.

D'Mello, R.J., Wass, A.M. (2012). Synergistic energy absorption in the axial crush response of filled circular cell honeycombs. Composite Structures 94(5): 1669-1676.

Ferri, E., Antinucci, E. He, M.Y., Hutchinson, J.W., Zok, F.W., Evans, A.G. (2006). Dynamic buckling of impulsively loaded prismatic cores. Journal of Mechanics of Materials and Structures 1(8): 1345-1365.

Goel, M.D. (2015). Deformation, Energy absorption and crushing behavior of single-, double- and multi-wall foam filled square and circular tubes. Thin-walled structures 90: 1-11.

Hanssen, A.H., Langseth, M., Hopperstad, O.S. (2000). Static and dynamic crushing of circular aluminum extrusions with aluminum foam filler. International Journal of Impact Engineering 24(5): 475-507.

Harte, A. Fleck, N. A., Ashby, M.F. (2000). Energy absorption of foam-filled circular tubes with braided composite walls. European Journal of Mechanics A/Solids 19(1): 31-50.

Kalhor, R., Case, S.W. (2015). The effect of FRP thickness on energy absorption of metal-FRP square tubes subjected to axial compressive loading. Composite Structures 130: 44-50.

Karagiozova, D. (2004). "Dynamic plastic" and "dynamic progressive" buckling of elastic-plastic circular shells - revisited. Latin American Journal of Solids and Structures 1: 423-441.

Kim, H. (2002). New extruded multi-cell aluminum profile for maximum crash energy absorption and weight efficiency. Thinwalled Structures 40(4): 311-327.

Lim, J., Bart-Smith, H. (2014). Theoretical approach on the dynamic global buckling response of metallic corrugated core sandwich columns. International Journal of Non-Linear Mechanics 65: 14-31.

Ronchietto, F., Chung Kim Yuen, S., Nurick, G. N. (2009). Response of axially stacked square tubes to axial impact loads. Latin American Journal of Solids of Structures 6: 413-440.

Santosa, S., Wierzbicki, T. (1998). Crash behavior of box columns filled with aluminum honeycomb or foam. Computers \& Structures 68: 343-367.

Santosa, S., Wierzbicki, T. (1999). The concept of double-walled sandwich columns for energy absorption. International Journal of Crashworthiness 4(2): 175-198.

Santosa, S., Wierzbicki, T., Hanssen, A.G., Langseth, M. (2000). Experimental and numerical studies of foam-filled structures. International Journal of Impact Engineering 24(5): 509-534.

Seitzberger, M., Rammerstorfer, F. G., Degischer, H. P., Gradinger, R. (1997). Crushing of axially compressed steel tubes filled with aluminum foam. Acta Mechanica 125: 93-105.

Seitzberger, M., Rammerstorfer, F. G., Gradinger, R., Degischer, H. P., Blaimschein, M., Walch, C. (2000). Experimental studies on the quasi-static axial crushing of steel columns filled with aluminum foam. International Journal of Solids and Structures 37(30): 4125-4147. 
Shahbeyk, S., Vafai, A., Petrinic, N. (2005). Axial crushing of metal foam-filled square columns - foam density distribution and impactor inclination effects. Thin-walled Structures 43(12): 1818-1830.

Stout, M.G. and Follansbee, P.S. (1986). Strain rate sensitivity, strain hardening, and yield behavior of 304L stainless steel. Journal of Engineering Materials and Technology 108: 344-353.

Sun, G., Li, G., Hou, S., Zhou, S., Li, W., Li, Q. (2010). Crashworthiness design for functionally graded foam-filled thin-walled structures Materials Science and Engineering A 527(7-8): 1911-1919.

Supian, A.B.M., Sapuan, S.M., Zuhri, M.Y.M., Syams, Z.E., Ya, H.H. (2018). Hybrid reinforced thermoset polymer composite in energy absorption tube application: A review. Defense Technology 14(4): 291-305.

Velmururugan, R., Muralikannan, R. (2009). Energy absorption characteristics of annealed steel tubes of various cross sections in static and dynamic loading. Latin American Journal of Solids and Structures 6: 385-412.

Wadley, H.N.G. (2006). Multifunctional periodic cellular metals, Philosophical Transactions of the Royal Society A 364: 31-68.

Wicks, N., Hutchinson, J.W. (2004). Performance of sandwich plates with truss cores. Mechanics of Materials 36: 739-751.

Wierzbicki, T., Abramowicz, W. (1983). On the crushing mechanics of thin-walled structures. Journal of Applied Mechanics 50(4a): 727-734.

Yan, L., Chouw, N., Jayaraman, K. (2014). Effect of triggering and polyurethane foam-filler on axial crushing of natural flax/epoxy composite tubes. Materials and Design 56: 528-541.

Yin, H. Wen, G., Liu, Z., Qing, Q. (2014). Crashworthiness optimization design for foam-filled multi-cell thin-walled structures. Thin-walled Structures 75: 8-17.

Zaeri, H., Kroger, M. (2008). Optimum honeycomb filled crash absorber design. Materials and Design 29(1): 193-204.

Zhang, G., Wang, B., Ma, L., Wu, L., Pan, S., Yang, J. (2014). Energy absorption and low velocity impact response of polyurethane foam filled pyramidal lattice core sandwich panels. Composite Structures 108: 304-310.

Zok, F.W., Rathbun, H., He, M., Ferri, E., Mercer, C., McMeeking, R.M., Evans, A.G. (2005). Structural Performance of metallic sandwich panels with square honeycomb cores, Philosophical Magazine 85(26-27): 11-21. 